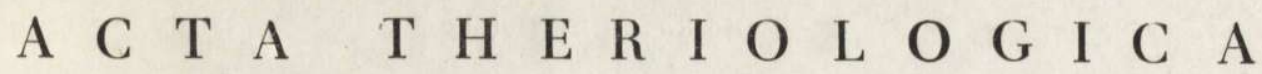 \\ VOL. XI, 7: 269-280.

\section{Ein Versuch zur Bestimmung der Grösse des Wirbelkanals beim Rothirsch}

[Mit 4 Tabellen und 2 Abb.]

\begin{abstract}
Die Autoren führten Kapazitätsmessungen der Wirbelkanäle bei 36 Hirschen (27 Hirschböcken und 9 Hirschkühen) durch, wobei sie sich der eigenen Methode bedienten, die in der Ausfüllung der Wirbellöcher der einzelnen Wirbel vermittels Schrot beruht. Diese Methode, die von den bisher angewandten Breiten- und Höhenmessungen der Wirbellöcher, eine grössere Präzision ermöglicht, erlaubt ausserdem des Ziehen von mehr weitgehenden Folgerungen im betreff der Grösse sowohl der entsprechenden Abschnitte des Wirbelkanals wie auch des Wirbelkanals als Ganzes. Sich dieser Methode bedienend - untersuchten die Autoren die postembryonale Entwicklung des Wirbelkanals und ausserdem bestimmten sie die Mitabhängigkeit zwischen der Grösse der Schädelhöhle und derfenigen des Wirbelkanals.
\end{abstract}

\section{EINFUHHRUNG}

Die Untersuchungen über den Wirbelkanal begrenzten sich bisher nur auf Breiten- und Höhenmessungen der Wirbellöcher, Foramina vertebralia (D u e r s t, 1926). deren Genauigkeit in Hinsicht auf die wenig stabilen Messungspunkte nicht genügend präzis ist. Mit diesem Problem befassten sich, jedoch nur marginal $\mathrm{Koch}$ (1932) und J a n icki (1937) während ihrer Untersuchungen über das Knochengerüst des Wisents, wobei sie Messungen der Wirbellöcher der einzelnen Wirbel durchgeführt haben. Der Wirbelkanal anderer Säugetiere, in betreff dessen was uns interessiert, ist nicht untersucht worden.

Der Gegenstand unserer Untersuchungen über die Kapazität des Wirbelkanals in Verbindung mit dem Ausarbeiten einer eigenen Methodik ist die Wirbelsäule des Hirsches, Cervus elaphus Linnaeus, 1758. 


\section{MATERIAL UND METHODE}

Das Untersuchungsmaterial bildeten 36 ausmazerierte Wirbelsäulen von Hirschen verschiedenen Alters darunter 29 Hirschböcke und 9 Hirschkühe, die sich im Osteologischen Museum des Institutes für Anatomie der Tiere der Landwirtschaftlichen Hochschule in Warszawa befinden. Die überwiegende Mehrzahl der den Untersuchungszwecken untergebenen Knochengerüste stammt von in den letzten Jahren (1959-1962) auf dem Gebiet von Polen abgeschossenen Hirschen. Man kann anrehmen, dass all diese Exemplare eine einheitliche Populationsform darstellen, die sich unter dem Einfluss von örtlichen ökologischen Bedingungen gestaltet hat, denn in den letzten Jahrzehnten wurden keine Hirsche aus dem Ausland nach Polen eingeführt (M y s t k ow s k a, 1966).

Das Material wurde in 4 Altersgruppen eingeteilt: das Tieralter wurde auf Grund des Zustandes des Gebisses bestimmt, wobei man sich auf die Arbeit von $\mathrm{H} \mathrm{a} \mathrm{be} \mathrm{r-}$ m e h 1 (1961) gestützt hat, aber auch auf Grund der Verhaltensstufe der Epiphysenfugenknorpel.

Zur I. Gruppe zählte man Individuen im Alter von 1-3 Jahren; zur II. - von \pm bis 7 Jahren; zur III. - von 8-12 Jahren und schliesslich zur IV. - oberhalb von 12 Jahren.

- Kapazitätsmessungen wurden vermittels Schrot Nr. 6 - Durchmesser 2,5 mm apart für jedes Wirbelloch der einzelnen Wirbel durchgeführt; eine Ausnahme bildeten die Kreuzbeine, deren Kapazität der Wirbelkanảle in ihrer Gesamtheit gemessen wurden. Die Vorbereitung der Wirbel zu Untersuchungszwecken beruhte auf dem Verkleben der kranialen Wirbellöcher mit Plastilin, aber auch in Abhängigkeit eines gewissen Abschnittes - der kranialen - und kaudalen Wirbelausschnitte, oder auch der Zwischenwirbellöcher. Das Wirbelloch der einzelnen Wirbel füllte man durch das kaudale Wirbelloch mit Schrot aus. Im Kreuzbein verklebte man den Hiatus caudalis Canalis sacralis und die Foramina sacralia dorsalia und die Formina sacralia ventralia, aber den Schrot führte man durch den Hiatus cramalis Canalis sacralis hinein.

Die Schrotmenge, die die Wirbellöcher und den Wirbelkanal des Kreuzbeines ausfüllte, wurde in einer kalibrierten Mensur bis zur Genauigkeit bis zu $0,5 \mathrm{~cm}^{3}$ gemessen. Die Messungen auf den Wirbeln aller Abschnitte der Wirbelsäule wurden zweimal durchgeführt und als Grundlage für weitere Erwägungen bediente man sich der Mittelwerte.

In einigen Fällen wurde die Kapazität auf unmazerierten Wirbelkanälen der kompletten Lendenabschnitte der Wirbelsäule gemessen und das erhaltene Ergebnis wurde mit der Summe derjenigen Messungen verglichen, die auf den einzelnen Wirbeln derselben Abschnitte durchgeführt worden waren. Es hat sich erwiesen, dass die Unterschiede hier minimal sind und in einem Falle z.B. mit dem Hirschbock (Katalog Nr. 367) betrug die Kapazität dieses Abschnittes als Ganzes gemessen $117 \mathrm{~cm}^{3}$, aber auf den einzelnen Wirbeln $-119 \mathrm{~cm}^{3}$; bei der Hirschkuh (Katalog Nr. 449) dementsprechend $65 \mathrm{~cm}^{3}$ und $66 \mathrm{~cm}^{3}$.

Zu Konfrontationszwecken der Mitabhängigkeit zwischen der Kapazität des Wirbelkanals und derjenigen der Schädelhöhle reichte man zu den Messungen der letzteren. Die Mittelwerte in den einzelnen Gruppen wurden auf Grund der eigenen Untersuchungen wie auch aus den, der Arbeit von M y s tkow s k a (1966) entnommenen, Angaben berechnet. Dabei zog man nử die Messungen der Kapazität der Schädelhöhlen derjeniger Individuen in Betracht, deren Wirbelkanäle ebenfalls Kapazitätsmessungen urterzogen worden waren. 
Für Fachausdrücke nahmen wir uns die Lehrbücher von Kolda (1936), P oplewski (1948) und Nickel-Schummer-Seiferle (1954) zum Vorbild.

\section{ANALYSE DER ERGEBNISSE}

\section{Pars cervicalis}

Der Halsabschnitt, Pars cervicalis, zählt in der Regel 7 Wirbel. Eine gewisse morphologische Besonderheit der ersten zwei Halswirbel kommt ebenfalls in der Kapazität ihrer Wirbellöcher zum Ausdruck. Der mit dem Schädel unmittelbar kontaktierende erste Wirbel kennzeichnet sich besonders durch die grosse Kapazität des Wirbelloches, die in Abhängigkeit von der Altersgruppe bei Hirschböcken von $23,5 \%$ bis $25,5 \%$ und bei Hirschkühen von $21,2 \%$ bis $23,6 \%$ der Kapazität des ganzen Wirbelkanals des Halsabschnittes ausmacht. Der prozentsatzmässige Anteil der Kapazität des Wirbelloches des zweiten Halswirbels ist entsprechend kleiner und schwankt bei Hirschböcken in den Grenzen von $14,4 \%$ bis $17,2 \%$ und bei Hirschkühen von 14,8 bis $16,6 \%$. Diese Kapazität ist die zweitgrösste (nach dem Kopfträger) unter den übrigen Halswirbeln.

Die Halswirbel von III bis VI weisen eine grosse individuelle Veränderlichkeit auf, wenn es sich um Kapazitätsmessungen der Wirbellöcher handelt und es fällt schwer in dieser Hinsicht eine gewisse Regelmässigkeit zu erfassen. Unter den besprochenen Wirbeln kennzeichnet sich der Wirbel VI durch die grösste Kapazität, aber auch hier kann man Ausnahmen antreffen (II. Gruppe, 우). Die Kapazitätsmessungen der übrigen Wirbel halten sich auf demselben Niveau, jedoch mit gewissen Schwankungen. Wirbel VII hat am öftesten eine kleinere Kapazität als Wirbel VI, aber seine Kapazität kann auch grösser sein (Tabelle 2). Wie es sich aus Abb. 1 ergibt, so kann der Mittelwert der Kapazität des Wirbelkanals des ganzen Halsabschnittes sowohl bei Hirschböcken wie auch bei Hirschkühen in Gruppe I am kleinsten sein. Bei Hirschböcken beträgt er $113,8 \mathrm{~cm},{ }^{3}$ bei Hirschkühen $125,3 \mathrm{~cm}^{3}$. In Gruppe II vergrössert er sich im Vergleich zu Gruppe I, bei Hirschböcken um 47,5\% und bei Hirschkühen um 13,6\% und beträgt dementsprechend $-165,8 \mathrm{~cm}^{3}$ und $142 \mathrm{~cm}^{3}$. In Gruppe III notiert man ebenfalls einen weiteren Anwuchs der Kapazität bei beiden Geschlechtern, die dementsprechend $-180,4 \mathrm{~cm}^{3}$ und $147,7 \mathrm{~cm}^{3}$ beträgt (bei Hirschböcken um $12,8 \%$ und bei Hirschkühen um 4,6\%). In Gruppe IV - Hirschböcke fällt die Kapazitätsmessung im Vergleich mit Gruppe III um $11,1 \%$ und beträgt $167,7 \mathrm{~cm}^{3}$, aber sie ist jedoch um $1,7 \%$ grösser als in Gruppe II; bei Hirschkühen dagegen weist die Kapazitätsmessung eine weitere Anwuchstendez auf (um 19,2\%) und beträgt $171,7 \mathrm{~cm}^{3}$.

Die Kapazität des Wirbelkanals des besprochenen Abschnittes bildet 


\begin{tabular}{|c|c|c|c|}
\hline 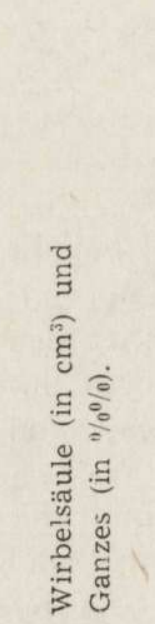 & 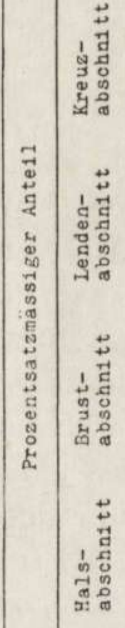 & 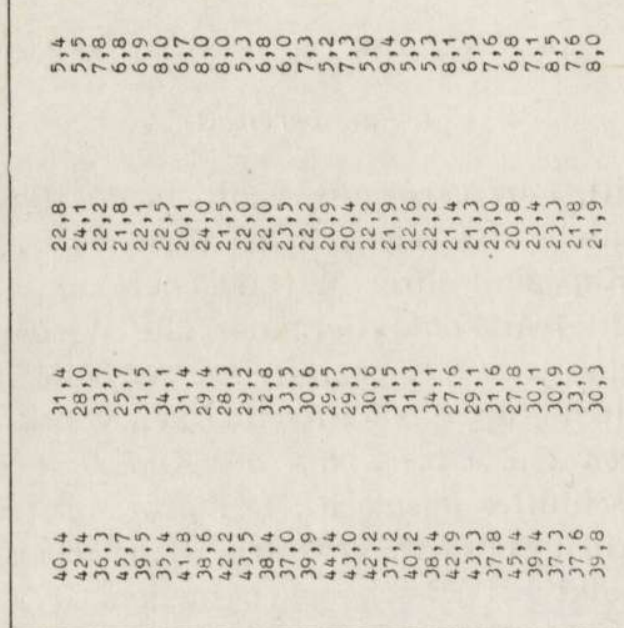 & 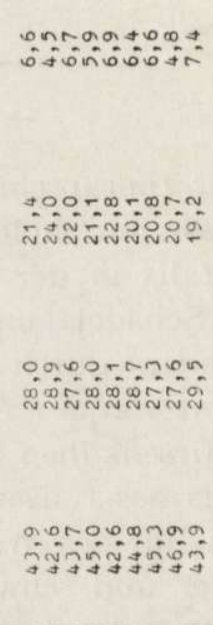 \\
\hline 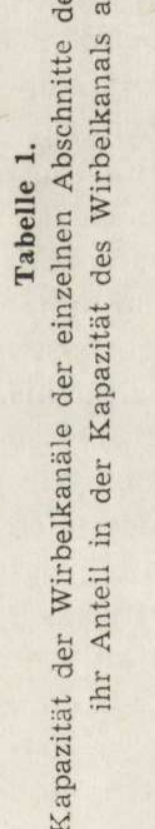 & 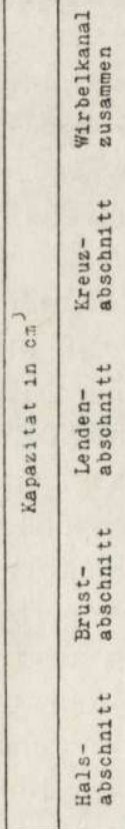 & 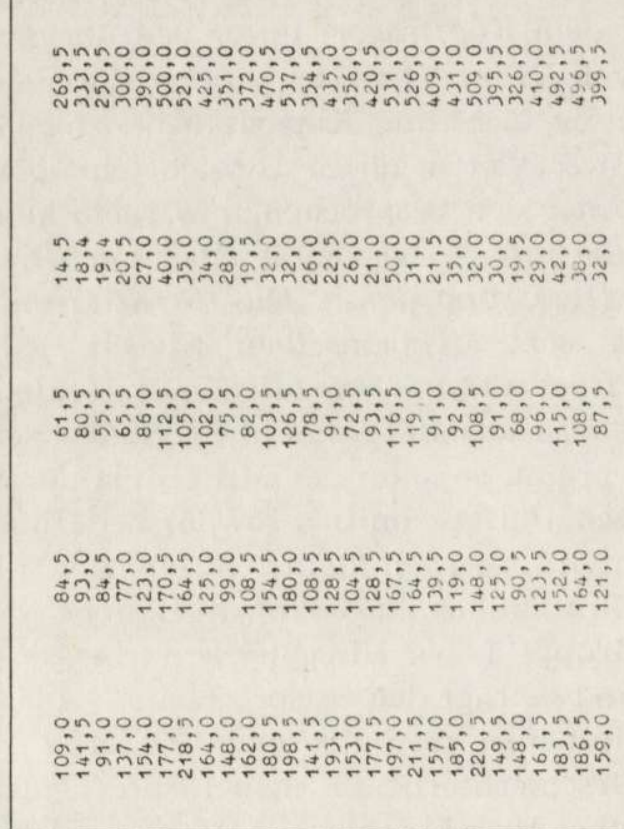 & 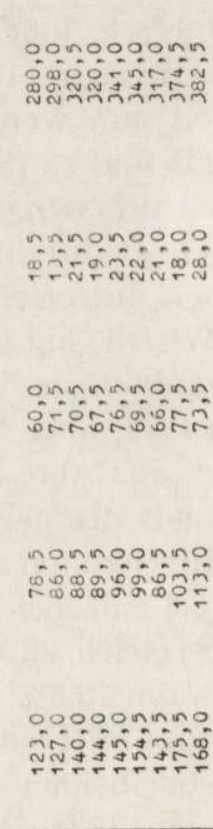 \\
\hline & 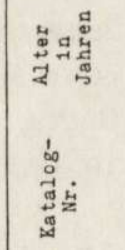 & 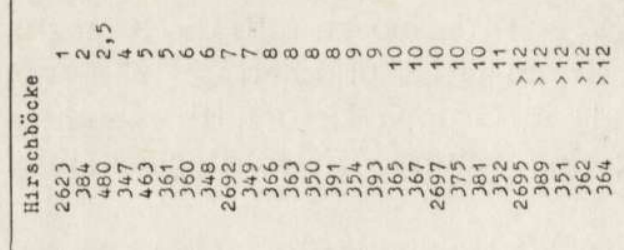 & 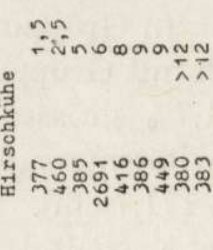 \\
\hline
\end{tabular}


den grössten Prozentsatz an Kapazität für den ganzen Wirbelkanal unter den übrigen Abschnitten der Wirbelsäule. Dieser Anteil beträgt bei Hirschböcken $35,4 \%-45,7 \%$; bei Hirschkühen sind diese Schwankungen kleiner - von $42,6 \%$ bis $46,9 \%$ (Tabelle 1 ).

Der Geschlechtsdimorphismus drückt sich bei Vergleich mit entsprechenden Gruppen nur in den Mittelgrössen der Kapazität der Wirbelkanäle des Halsabschnittes aus.

Tabelle 2.

Mittelwerte der Kapazität der Wirbellöcher der einzelnen Wirbel (in $\mathrm{cm}^{3}$ ) der entsprechenden Altersgruppen.

\begin{tabular}{|c|c|c|c|c|c|c|c|c|c|}
\hline \multirow{2}{*}{\multicolumn{2}{|c|}{ Gruppe }} & \multicolumn{4}{|c|}{ H1rschböcke } & \multicolumn{4}{|c|}{ H1rsobkühe } \\
\hline & & I & II & III & IV & I & II & III & IV \\
\hline \multirow{8}{*}{ 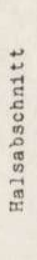 } & $y_{1} \mathrm{rbel}$ & & & & & & & & \\
\hline & I & 29,0 & 41,2 & 44,2 & 39,4 & 29,5 & 30,5 & 34,8 & 36,5 \\
\hline & II & 16,5 & 25,5 & 28,1 & 28,8 & 18,5 & 23,0 & 22,2 & 28,5 \\
\hline & III & 13,5 & 19,1 & 21,5 & 19,9 & 14,7 & 19,0 & 17,8 & 21,5 \\
\hline & IV & 13,3 & 19,3 & 21,2 & 19,7 & 15,2 & 18,5 & 17,8 & 21,2 \\
\hline & $v$ & 13,0 & 20,0 & 21,8 & 19,4 & 15,2 & 17,5 & 17,8 & 20,2 \\
\hline & VI & 14,0 & 21,2 & 22,8 & 20,5 & 16,0 & 17,2 & 18,8 & 21,7 \\
\hline & VII & 14,5 & 19,4 & 20,7 & 19,9 & 15,7 & 16,2 & 18,3 & 22,0 \\
\hline \multirow{13}{*}{ 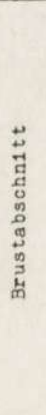 } & I & 10,0 & 13,5 & 15,4 & 14,2 & 10,7 & 10,5 & 12,3 & 14,2 \\
\hline & II & 7,7 & 10,8 & 12,8 & 11,0 & 8,2 & 8,5 & 8,8 & 10,5 \\
\hline & III & 6,8 & 10,6 & 12,0 & 11,1 & 7,0 & 7,2 & 7,8 & 9,2 \\
\hline & IV & 6.7 & 10,0 & 11,5 & 11,0 & 6,7 & 6,7 & 7,3 & 8,5 \\
\hline & $\nabla$ & 6,3 & 9,5 & 10,8 & 10,3 & 6,0 & 6,5 & 6,8 & 8,0 \\
\hline & VI & 6,0 & 9,3 & 10,0 & 9,9 & 5,5 & 6,5 & 6,3 & 7,5 \\
\hline & VII & 6,0 & 8,7 & 9,4 & 9,3 & 5,5 & 6,2 & 5,8 & 7,2 \\
\hline & VIII & 5,7 & 8,2 & 8,9 & 8,5 & 5,0 & 5,7 & 5,8 & 6,7 \\
\hline & IX & 5,7 & 7,8 & 8,2 & 8,2 & 5,0 & 5,5 & 5,7 & 6,7 \\
\hline & $x$ & 5,7 & 7,5 & 8,2 & 7,9 & 4,7 & 5,2 & 5,5 & 6,5 \\
\hline & $X I$ & 6,3 & $8, C$ & 8,8 & 8,5 & 5,0 & 6,0 & 6,0 & 6,7 \\
\hline & XII & 7,2 & 9,5 & 10,6 & 9,9 & 5,7 & 6,7 & 7,2 & 7,4 \\
\hline & XIII & 7,3 & 10,5 & 11,3 & 10,4 & 7,0 & 7,5 & 8,0 & 8,5 \\
\hline \multirow{6}{*}{ 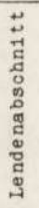 } & $I$ & 8,2 & 11,1 & 12,5 & 11,7 & 8,0 & 8,7 & 9,0 & 9,2 \\
\hline & II & 8,8 & 12,4 & 13,8 & 12,9 & 8,5 & 9,2 & 9,7 & 9,7 \\
\hline & III & 9,5 & 13,3 & 14,8 & 14,0 & 9,5 & 10,2 & 10,7 & 11,0 \\
\hline & IV & 11,2 & 15,0 & 16,6 & 15,9 & 10,5 & 11,7 & 12,2 & 12,7 \\
\hline & $\mathrm{V}$ & 14,3 & 18,4 & 20,4 & 19,8 & 13,5 & 14,5 & 14,8 & 16,0 \\
\hline & vI & 13,8 & 19,5 & 20,5 & 20,6 & 13,7 & 14,5 & 14,3 & 16,7 \\
\hline
\end{tabular}

\section{Pars thoracalis}

Den am häufigsten 13 Wirbel zählenden Brustabschnitt, Pars thoraca. lis, kennzeichnet eine grosse Beständigkeit, wenn es sich um die Kapa. zität der Wirbellöcher der einzelnen Wirbel handelt (Tab. 2). Derjenige Wirbel, der die grösste Kapazität besitzt, ist in der Regel der Wirbel I ohne Rücksicht auf Altersgruppe und Geschlecht. Von diesem Wirbel ab 


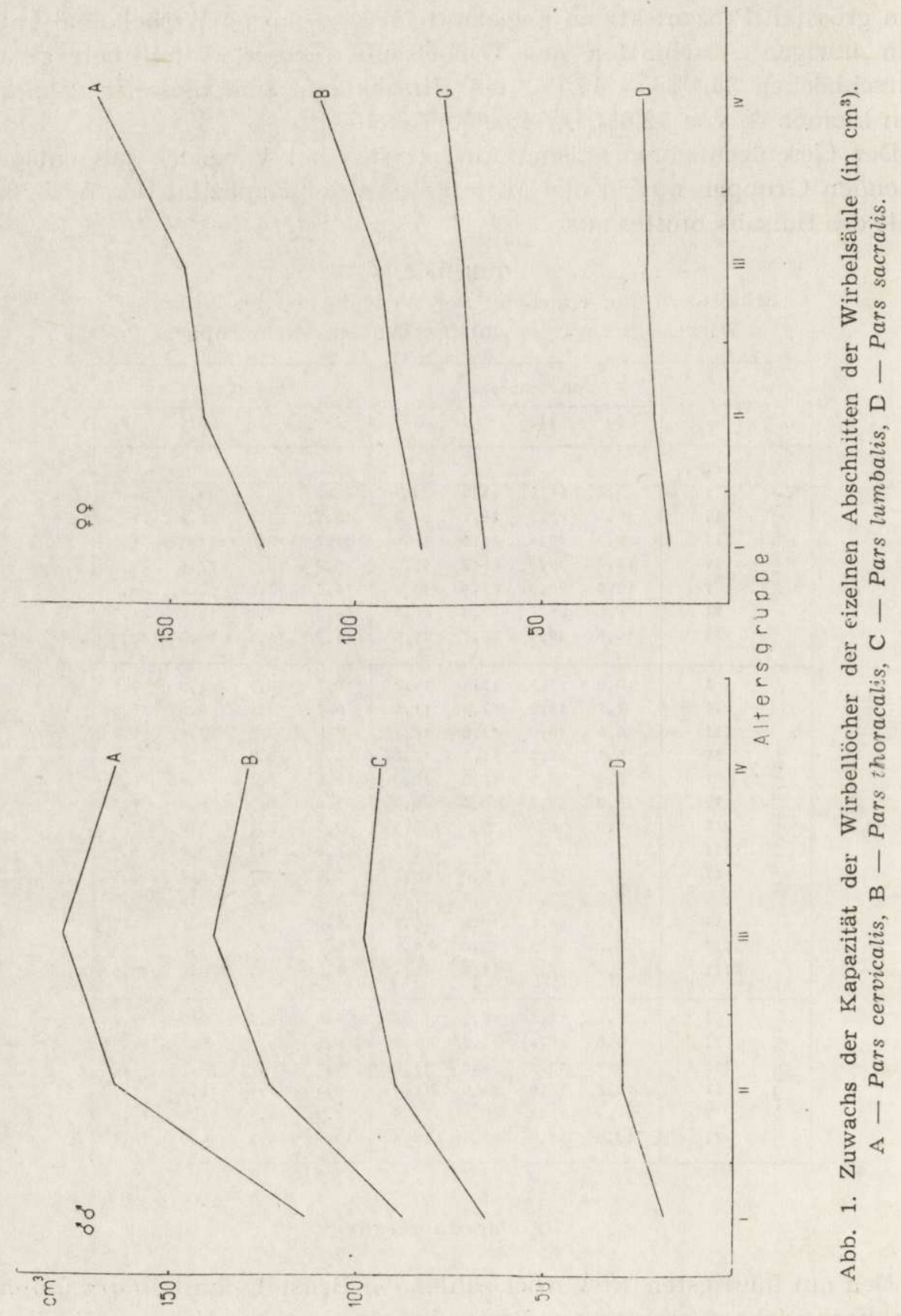


erfolgt eine stufenweise Verminderung dieser Messung, die sich bis zu Wirbel X langzieht. Bei Wirbel XI findet wieder ein gewisser, wenn auch ungrosser Anwuchs statt, der sich ebenfalls in den nachfolgenden Wirbeln offenbart, das ist in XII und XIII.

Der Wirbelkanal des ganzen Brustabschnittes hat bei Hirschböcken in der Gruppe I durchschnittlich eine Kapazität von $87,3 \mathrm{~cm}^{3}$. Diese Kapazität vergrössert sich nachfolgend in Gruppe II um 42,0\% und beträgt durchschnittlich $124,0 \mathrm{~cm}^{3}$. Diese Messung hat wiederum in Gruppe III eine Anstiegstendez und beträgt $139,0 \mathrm{~cm}^{3}$, was im Verhältnis zur Gruppe II einen Anwuchs um 17,2\% ausmacht. Erwähnenswert ist diese T'atsache, dass Gruppe IV schon eine um 10,1\% kleinere Messung hat als Gruppe III; sie beträgt durchschnittlich $130,2 \mathrm{~cm}^{3}$ (Abb. 1).

Bei Hirschkühen wächst die Kapazität des Wirbelkanals des besprochenen Abschnittes stufenweise von Gruppe I bis Gruppe IV an, und die durchschnittlichen Messungen gestalten sich folgendermassen: in Gruppe I - 82,2 $\mathrm{cm}^{3}$; in Gruppe II $-89,0 \mathrm{~cm}^{3}$ (Anwuchs um $8,3 \%$ ); in Gruppe III - 93,8 $\mathrm{cm}^{3}$ (Anwuchs um 5,8\%) und schliesslich in Gruppe IV $108,2 \mathrm{~cm}^{3}$ (Anwuchs um 17,5\%) - Abb. 1.

Der prozentsatzmässige Anteil der Kapazität des Wirbelkanals des Brustabschnittes schwankt in der Kapazität des ganzen Wirbelkanals bei Hirschkühen, unabhängig vom Alter, in den Grenzen von $27,3 \%$ bis $29,5 \%$; bei Hirschböcken sind diese Schwankungen grösser und er beträgt von $25,7 \%$ bis $34,1 \%$ (Tab. 1 ).

Der sich in den Mittelwerten der Kapazitätsmessungen des Wirbelkanals im Brustabschnitt manifestierende Geschlechtsdimorphismus lässt keine gänzliche Trennung des Geschlechtes bei der Untersuchung der einzelnen Individuen $\mathrm{zu}$, und zwar aus Gründen der individuellen Veränderlichkeit. Eine Ausnahme bildet nur Gruppe III, in der die Messungen der Hirschböcke diejenigen der Hirschkühe bedeutend übersteigen.

\section{Pars lumbalis}

Der Lendenabschnitt, Pars lumbalis, der aus 6 Wirbeln besteht, weist ebenfalls eine grosse Beständigkeit auf, wenn es sich um Kapazitäten der Wirbellöcher der einzelnen Wirbel handelt. Die kleinste Kapazität stellte man in Wirbel I fest, von dem ab ein ständiger, stufenweiser Anwuchs dieser Messung beginnt und der meistenteils bis zum letzten Wirbel andauert. Diesen Zustand notierte man in den Hirschbockgruppen II, III und IV und in der Gruppe I und Gruppe IV bei Hirschkühen. In den übrigen Gruppen hat der letzte sechste Lendenwirbel entweder eine kleinere Kapazität des Wirbelloches als im vorhergehenden Wirbel (Gruppe I Hirschböcke und Gruppe III - Hirschkühe) oder auch eine gleiche (Gruppe II - Hirschkühe). Es muss aber unterstrichen werden, dass die 
sogar kleinere als im Wirbel V Kapizität des letzten Wirbelloches durch ihre Grösse die Wirbelmessungen der übrigen überragt (Tab. 2).

Die Summe der Kapazitätsmessungen der Wirbellöcher der Lendenwirbel, die die Kapazität des Wirbelkanals des ganzen besprochenen $\mathrm{Ab}$ schnittes ausmacht, ist bei Hirschböcken in Gruppe I am kleinsten und beträgt durchschnittlich $65,8 \mathrm{~cm}^{3}$. In Gruppe II erfolgt im Vergleich mit Gruppe I ein Anwuchs um 36,5\% und die Mittelmessung beträgt $39,8 \mathrm{~cm}^{3}$. In Gruppe III notiert man ebenfalls eine Anwuchstendenz gegenüber Gruppe II (um 13,3\%) - die Mittelmessung beträgt $98,6 \mathrm{~cm}^{3}$. Gruppe IV hat, ähnlich wie dies in den vorigen Abschnitten der Fall war, eine Sen-
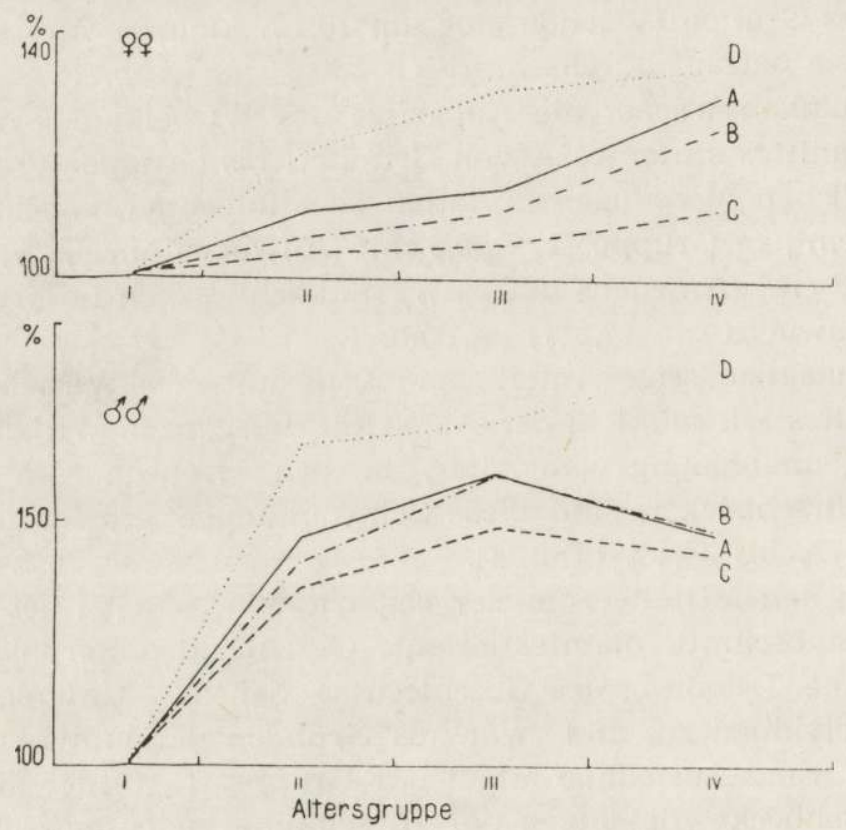

Abb. 2. Prozentsatzmässiges Zuwachs der Kapazität der Wirbellöcher der einzelnen Abschnitten der Wirbelsäule.

A - Pars cervicalis, B - Pars thoracalis, C - Pars lumbalis, D - Pars sacralis.

kung des Mittelmessungswertes im Vergleich mit Gruppe III (um 5,6\%) bis $94,9 \mathrm{~cm}^{3}$. Im Lendenabschnitt überragt auch die Mittelmessung der Kapazität der Gruppe IV die Messungsgrösse von Gruppe II, wenn sie auch kleiner ist von Gruppe III (Abb. 1).

Bei Hirschkühen wächst die Kapazịtätsmessung des Wirbelkanals des Lendenabschnittes mit verschiedener Intensität von Gruppe I bis zur Gruppe IV. Die Mittelmessungen für die einzelnen Gruppen stellển sich folgendermassen dar: Gruppe I - 65,7 $\mathrm{cm}^{3}$; Gruppe II - 69,0 $\mathrm{cm}^{3}$ (Anwuchs um 5,0\%); Gruppe III - 70,7 $\mathrm{cm}^{3}$ (Anwuchs um 2,6\%) und schlic sslich Gruppe IV - 75,5 $\mathrm{cm}^{3}$ (Anwuchs um 7,3\%) - Abb. 1, 2. 
Die Kapazität des Wirbelkanals des Lendenabschnittes macht bei Hirschböcken $20,1 \%-24,1 \%$ und bei Hirschkühen $19,2 \%-24,0 \%$ der Kapazität des ganzen Wirbelkanals aus (Tab. 1).

Den Geschlechtsdimorphismus kann man bei der Vergleichung der Mittelmessungen der entsprechenden Gruppen feststellen.

Tabelle 3.

Mittelwerte der Kapazität der Wirbelkanäle und ihr Anwuchs in den einzelnen Altersgruppen.

\begin{tabular}{|c|c|c|c|c|c|c|c|c|}
\hline \multirow[t]{2}{*}{ Gruppe } & \multicolumn{2}{|c|}{1} & \multicolumn{2}{|c|}{ II } & \multicolumn{2}{|c|}{ III } & \multicolumn{2}{|c|}{ IV } \\
\hline & $d^{\prime}$ & $q$ & 0 & $q$ & $\sigma$ & $q$ & $\sigma$ & $q$ \\
\hline $\begin{array}{l}\text { Kapazität } 1 \mathrm{~m} \text { M1ttel } \\
\text { des W1rbelkanals } 1 \mathrm{n} \mathrm{cm}^{3}\end{array}$ & 284,5 & 289,0 & 408,7 & 320,2 & 447,9 & 334,3 & 424,9 & 378,5 \\
\hline $\begin{array}{l}\text { Unterschiede 1m } \\
\text { Anwuchs } 1 \mathrm{n}^{3}\end{array}$ & - & - & 124,2 & 31,2 & 39,2 & 14,1 & $-23,0$ & 44,2 \\
\hline $\begin{array}{l}\text { Kapaz1tät } 1 \text { m M1ttel } \\
\text { des N1rbelkanals in } \varnothing\end{array}$ & 100,0 & 100,0 & 143,6 & 110,8 & 157,4 & 115,7 & 149,3 & 131,0 \\
\hline $\begin{array}{l}\text { Unterschied it } \\
\text { Anwuchs in } \varnothing\end{array}$ & - & - & 43,6 & 10,8 & 13,8 & 4,9 & $-8,1$ & 15,3 \\
\hline
\end{tabular}

Tabelle 4.

Gestaltung des Koeffizienten: Kapazität des Wirbelkanals in $\mathrm{cm}^{3}$ zur Kapazität der Schädelhöhle in $\mathrm{cm}^{3}$.

\begin{tabular}{|l|c|c|c|c|}
\hline Gruppe & I & II & III & IV \\
\hline H1rschböcke & $1: 1,15$ & $1: 0,91$ & $1: 0,84$ & $1: 0,77$ \\
H1rschkühe & $1: 1,23$ & $1: 1,00$ & $1: 0,93$ & $1: 0,98$ \\
\hline
\end{tabular}

4. Pars sacralis

Das Auftreten von $4-5$ in das Kreuzbein, Os sacrale, verwachsenen Wirbeln verursacht, dass die Kapazität des Wirbelkanals dieses Knochens eine beträchtliche individuelle Veränderlichkeit aufweist (Tab. 1). Die Mittelwerte der einzelnen Gruppen dagegen kennzeichnen einen dauernden im verschiedenen Grade vom Alter abhängigen Anwuchs von Gruppe I bis Gruppe IV und dies sowohl bei Hirschböcken wie auch Hirschkühen (Abb. 1). Die durchschnittliche Kapazität des Wirbelkanals des Kreuzbeines in Gruppe II der Hirschböcke ist um $66,3 \%$, aber bei Hirschkühen um $26,2 \%$ grösser im Vergleich mit Gruppe I. In Gruppe III 
notiert man einen weiteren Anwuchs - demensprechend um 4,5\% und $12,5 \%$; ähnlich wie auch in Gruppe IV - um $12,6 \%$ und $5,0 \%$.

Der prozentsatzmässige Anteil des Wirbelkanals des Kreuzbeines in der Kapazität des ganzen Wirbelkanals schwankt in folgenden Grenzen: bei Hirschböcken von $5,0 \%$ bis $9,4 \%$, bei Hirschkühen von $4,9 \%$ bis $7,4 \%$ (Tab. 1).

Die grosse individuelle Veränderlichkeit in der Kapazität der Wirbelkanäle der Kreuzbeine lässt keine Trennung des Geschlechtes zu.

Der Wirbelkanal als ganzes hat die kleinste Kapazität in Gruppe I. Der mit dem Alter verbundene Anwuchs der Kapazität findet bei Hirschkühen in allen untersuchten Gruppen statt. Bei Hirschböcken erhält sich die Anwuchstendenz nur in den drei ersten Gruppen (Tabelle 3).

Interessant sieht die Zusammenstellung der Kapazität des Wirbelkanals mit der Kapazität der Schädelhöhle aus (Tabelle 4). Dieser Koeffizient weist bei Hirschböcken eine deutliche Tendenz des Sinkens auf, die mit dem Alter verbunden ist. Er beträgt in Gruppe I - 1:1,15 und dies beweist, dass die Schädelhöhle eine grössere Kapazität hat als der Wirbelkanal. In Gruppen II, III und IV fällt der Anwuchswert für die Schädelhöhle stufenweise und ist kleiner als für den Wirbelkanal. Bei Hirschkühen formiert sich dieser Koeffizient ähnlich wie bei Hirschböcken, aber der Anwuchs der Kapazität der Schädelhöhle gestaltet sich in den Gruppen II, III und IV als gleich oder er ist ein wenig kleiner vom Anwuchs der Kapazität des Wirbelkanals.

Der Geschlechtsdimorphismus offenbart sich in den Mittelmessungen der Kapazität des Wirbelkanals der entsprechenden Gruppen.

Der Mittelwert von Gruppe IV ist bei Hirschkühen kleiner als der Mittelwert von Gruppe II bei Hirschböcken (Tab. 3).

\section{FOLGERUNGEN}

Die Untersuchungen der Kapazität des Wirbelkanals scheinen bei der Bestimmung der Grösse dieses Kanals eine gute Methode zu sein. Die Art und Weise der Durchführung der Messungen sind nicht kompliziert, aber gleichzeitig entsprechender für eine Höhle mit natürlicher Kanalgestaltung. Die bisher angewandten Messungen der Breite und Höhe der Wirbellöcher ermöglichen, ausser der in der Einführung signalisierten kleinen Präzision, kein Ziehen von mehr eingehenden Folgerungen in betreff der Grösse der entsprechenden Abschnitte des Wirbelkanals wie auch des Wirbelkanals als Ganzes. Die Vergleichung der Entwicklung beider Höhlen für das zentrale Nervensystem war ebenfalls unmöglich. 
Die Kapazitätsmessungen geben eine Antwort auf das erwähnte Problem und ermöglichen ausserdem Beobachtungen über die postembryonale Entwicklung des Wirbelkanals und liefern ebenfalls mehr eingehende Angaben für die Konfrontation der Grösse sowohl der Wirbellöcher wie auch der Kanäle der einzelnen Abschnitte der Wirbelsäule.

Sich der Kapazitätsmessung des Wirbelkanals bedienend, gelang es aus auf einem verhältnismässig grossen Material von Hirschböcken die Regelmässigkeit der Verminderung beider Höhlen des zentralen Nervensystems bei den ältesten Individuen beiderlei Geschlechtes festzustellen, was durch vorher bestätigte Untersuchungen über die Kapazität aber nur für die Schädelhöhle des Hirsches festgestellt worden ist (M y s tk owska, 1966). Eine verhältnismässig kleine Anzahl an Hirschkühen im unseren Untersuchungsmaterial berechtigt uns nicht zum Ziehen von weitgehenden Folgerungen. Der weitere Anwuchs der Kapazität des Wirbelkanals bei den ältesten Hirschkühen jedoch findet in den Untersuchungen über die Kapazität der Schädelhöhle beim Hirsch ebenfalls eine Bestätigung der oben zitierten Autorin.

Zum Schluss ist es noch erwähnenswert, dass die Beobachtungen über die Kapazität des Wirbelkanals, die noch durch Längenmessungen der Wirbelkörper ergänzt worden sind, die Ausbreitung des Kanals für charakteristische Anschwellungen des Rückenmarkes lokalisieren dürfen.

\section{SCHRIFTTUM}

1. Duerst J. U., 1926: Vergleichende Untersuchungsmethoden am Skelett bei Säugern. (Abderhalden: Handbuch d. biol. Arbeitsmeth.), 7: 125-530. UrbanSchwarzenberg. Berlin-Vien.

2 Habermehl K. H., 1961: Die Altersbestimmung bei Haustieren, Pelztieren und beim jagdbaren Wild.: 1-223. Paul Parey. Berlin-Hamburg.

3. J a n i cki S., 1938: Badania nad szkieletem żubra (Bison bonasus L.). Prace Rolniczo-Leśne P.A.U., 27: 1-55. Kraków.

4. Koch W., 1932: Über Wachstums- und Altersveränderungen am Skelett des Wisents. Abh. Mat.-nุaturw. Abt., Bayer. Akad. W.iss. Suppl.-Bd., 15 Abh.: 555678. München.

5. Kolda J., 1936: Srovnavaci anatomie zvirat domacich. Tiskla Novina: 1-914. Brno.

6. Mystkowska E. T., 1966: Morphological variability of the skull and body weight of the red deer. Acta theriol. 11, 5: 129-194.

7. Nickel-Schummer-Seiferle 1954: Lehrbuch der Anatomie der Haustiere. Paul Parey, 1: 1-502. Berlin-Hamburg.

8. Poplewski R., 1948: Anatomia ssaków. 2: 1-690. Czytelnik. Stockholm.

Received February 3, 1965.

Institut für Anatomie der Tiere

an der Landwirtschaftliche Hochschule,

Warszawa, ul. Grochowska 272. 
Tadeusz ROSKOSZ i Stanisław PYTEL

\section{PRÓBA OKREŚLENIA WIELKOSCI KANAŁU KRĘGOWEGO U JELENIA NIZINNEGO}

\section{Streszczenie}

Autorzy dokonali pomiarów pojemności kanałów kręgowych 36 jeleni (27 samców i 9 samic), posługując się metodą własną, polegającą na wypełnianiu śrutem otworów kręgowych poszczególnych kręgów. Metoda ta poza większą precyzyjnością, od stosowanych dotychczas pomiarów szerokościowo-wysokościowych otworów kręgowych, pozwala na wyciąganie bardziej szczegółowych wniosków, dotyczących wielkości, zarówno odpowiednich odcinków kanału kręgowego, jak i kanału kręgowego jako całości.

Autorzy prześledzili rozwój pəstnatalny kanału kręgowego oraz ustalili współzależność między wielkością jamy czaszkowej a wielkością kanału kręgowego. 